\title{
The prognostic significance of $\beta$ human chorionic gonadotrophin and its metabolites in women with cervical carcinoma
} Robin A F Crawford, Ray K Iles, P G Carter, Carole J Caldwell, John H Shepherd,
Tim Chard
However, the clinical use of free $\beta$ hCG as a Aims-To examine long term survival of women with primary and recurrent cervical carcinoma in relation to (1) excretion of $\beta$-core (a urinary metabolite of $\beta$ human chorionic gonadotrophin ( $\beta$ hCG)) and (2) BhCG immunostaining of the tumours, to determine the suitability of these markers for assessing prognosis.

Methods-This was a prospective observational study undertaken in a gynaecological oncology centre: 57 women with primary cervical cancer and 42 with recurrent disease were recruited between January 1990 and September 1992. Kaplan-Meier survival analysis with the log rank test was used to assess survival differences with survival rate given per year of follow up.

Results-In primary disease, the four year survival for the $\boldsymbol{\beta}$-core negative group was $79 \%$, compared with $14 \%$ for the $\beta$-core positive group $(p=0.001)$. This was still significant for early stage disease or squamous lesions alone. In recurrent disease, $\beta$-core positivity was not prognostically significant. Immunohistochemistry was of no prognostic significance in either group.

Conclusions- $\beta$-core excretion appears to be useful in assessing prognosis of primary cervical cancer but not of recurrent disease. A large prospective study of urinary $\beta$-core in early stage cervical cancer is needed to determine whether it can be used as an index for modifying treatment.

(f Clin Pathol 1998;51:685-688)

Keywords: cervical cancer; $\beta$ human chorionic gonadotrophin; $\beta$-core; immunohistochemistry

Human chorionic gonadotrophin (hCG) is a valuable tumour marker for trophoblastic disease and germ cell tumours. In addition, synthesis of free $\beta$ hCG and its subunits by pelvic carcinomas such as those of the colon, ${ }^{1}$ urinary tract, ${ }^{2}$ prostate, ${ }^{3}$ uterus, ${ }^{4}$ and vulvovagina $^{5}$ has been reported. The presence of increased serum levels of hCG and its metabolites is generally agreed to be a sign of a poor prognosis $^{6-8}$ and it has been suggested that $\beta$ hCG might directly modify the growth of the cancer, leading to a worse outcome. ${ }^{6}$ Furthermore, positive immunohistochemical staining for $\beta$ hCG is also associated with a survival disadvantage following radiotherapy. ${ }^{9}$ tumour marker has been limited to a small number of patients owing to a short half life and rapid renal clearance. Attention has therefore been focused on urine metabolites, in particular $\beta$-core, ${ }^{10}$ which is produced as a result of the intrarenal metabolism of $\beta$ hCG. The majority of hCG immunoreactivity in urine is accounted for by $\beta$-core. Excess $\beta$-core is associated with gynaecological cancer (reviewed by Cole et $\left.a l^{4}\right)$. In a previous study from our group, ${ }^{11}$ we reported that $\beta$-core was a significant prognostic indicator for premenopausal women with primary cervical cancer followed up for only 18 months.

We now report the long term outcome of a group of patients with primary and recurrent of $\beta$-core. Some of these patients were included in our previous report. ${ }^{11}$ In addition, we report the results of immunohistochemical staining of the tumours for $\beta$ hCG.

\section{Methods}

The study included consecutive patients presenting with cervical cancer (primary and recurrent) at St Bartholomew's Hospital and the Royal Marsden Hospital between January 1990 and September 1992. Local research ethics committees had approved the protocol. Fifty seven women with primary disease and 42 with recurrent disease were identified. All patients with recurrence had already received pelvic radiotherapy. A representative block was selected for each patient from archival tissue and $3 \mu \mathrm{m}$ sections were mounted on coated slides for immunohistochemistry. An adjacent slide was stained with haematoxylin and eosin for reference. The histological diagnosis was reviewed by CJC.

BhCG

Samples suitable for immunohistochemistry were available from 46 patients with primary cervical cancer and from 29 with recurrent disease. For immunohistochemistry, sections were dewaxed and then pressure cooked for four minutes at $103 \mathrm{kPa}^{12}$ as the method of antigen enhancement. A rabbit polyclonal anti- $\beta$ hCG antibody (Dako, High Wycombe, UK) was used at a dilution at $1 / 5000$ overnight at $4^{\circ} \mathrm{C}$. A standard indirect avidin-biotin immunoperoxidase technique was then applied. A section of placenta was used a positive control, and the primary antibody was substituted with nonspecific rabbit antibody as negative control. cervical cancer in relation to urinary excretion
Accepted for publication 3 March 1998 


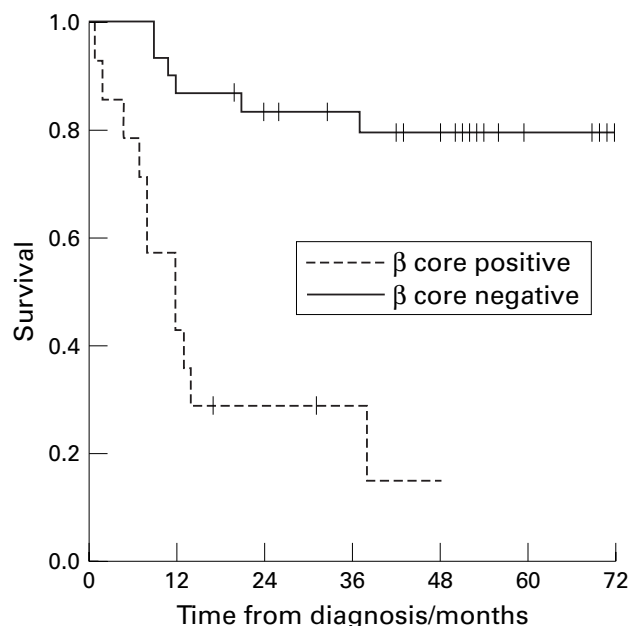

Figure 1 Kaplan-Meier survival curve for patients presenting with primary cervical cancer separated on the basis of the urinary $\beta$-core measured at presentation ( $\log$ rank test: $\left.\chi_{d=1}^{2}=19.74 ; p=0.001\right)$. $\beta$-Core negative group: $n=30$; six events; five year survival $79 \%$. $\beta$-Core positive group: $n=14 ; 11$ events; median survival 12 months, four year survival $14 \%$.

The slides were scored visually by two independent observers (RAFC, CJC) for granular cytoplasmic staining. The case was scored positive if any tumour cell showed positive staining.

$\beta$-CORE ESTIMATION

All women provided a urine sample at their initial attendance. Specimens with a creatinine concentration greater than $4 \mathrm{mmol} /$ litre were assayed for $\beta$-core as described ${ }^{11}$; values were considered raised if they were above the 90th centile for the relevant group (premenopausal $0.034 \mathrm{ng} \beta$-core $/ \mathrm{ml} / \mathrm{mmol}$ creatinine; postmenopausal $0.094 \mathrm{ng} \beta$-core $/ \mathrm{ml} / \mathrm{mmol}$ creatinine). Forty six women with primary disease and 38 women with recurrent cervical cancer had sufficiently concentrated urine for $\beta$-core estimation.

\section{PATIENT CHARACTERISTICS}

\section{Primary cervical cancer}

The median age of the group was 43 years (range 23 to 82 years) and 35 women were premenopausal. There was no age related survival advantage in this group of women comparing young women ( $<40$ years) with older women, nor any relation to menopausal status. There were 28 cases with stage 1 disease, 11 with stage 2,10 with stage 3 , five with stage 4 , and no record in three cases. Forty cases were squamous cell carcinoma, eight were adenocarcinoma, and seven had adenosquamous lesions (identified with periodic acid Schiff and alcian blue (PAS/AB) staining in addition to $\mathrm{H} \& \mathrm{E})$. In two cases the pathology was not recorded. The median time to death or date of census (April 1996) was 35 months (range 1 to 74 months). Follow up information was not available in two cases.

\section{Recurrent cervical cancer}

The median age of this group was 45 years (range 25 to 78 years). The median survival from initial diagnosis of cancer was 22 months

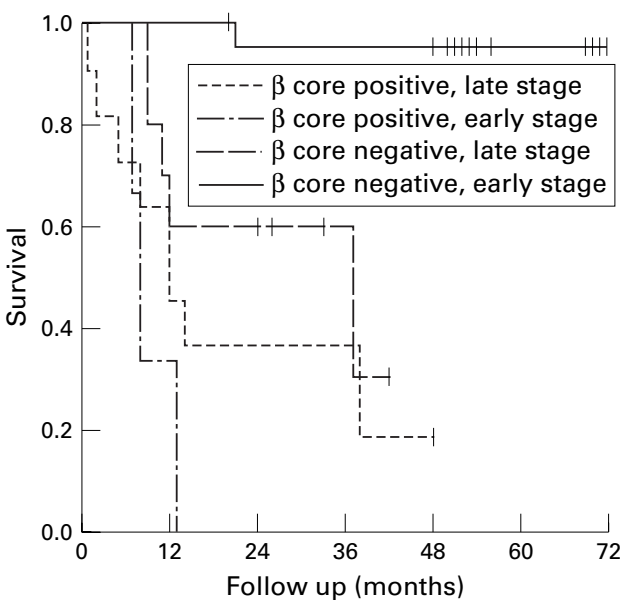

Figure 2 Kaplan-Meier survival curve for patients presenting with primary cervical cancer separated on the basis of $\beta$-core and stage (log rank test: $\chi^{2}=28.52 ; p=$ $0.001)$. $\beta$-Core negativelearly stage: $n=20$; one event; five year survival $95 \%$; $\beta$-core negative/late stage: $n=10$; five events; median survival 37 months. $\beta$-Core positivelearly stage: $n=3$; three events; median survival eight months; $\beta$-core positive/late stage: $n=11$; eight events; median survival 12 months.

(range 2 to 149 months). At initial diagnosis, there were 19 cases with stage 1 disease, 13 with stage 2 , five with stage 3 , four with stage 4 , and no record of initial stage in one case. Thirty cases were squamous cell carcinoma, five were adenocarcinoma, and five had adenosquamous lesions. The original histology was not recorded in one case. The median survival time from diagnosis of recurrent cancer, when the urine marker was investigated, was eight months (range 1 to 71 months). The five year survival of this group from presentation with recurrent cervical cancer was $10 \%$.

\section{STATISTICS}

The percentage survival for each group was calculated using the Kaplan-Meier method and comparisons between groups were performed using the log-rank test (SPSS for Windows, 6.1.3 1995). Where data did not extend to five years, survival rate is given per year of follow up in that group.

\section{Results}

PRIMARY DISEASE

Thirty one patients were $\beta$-core negative and 15 were $\beta$-core positive. The four year survival for women who were $\beta$-core negative was $79 \%$; the four year survival of the $\beta$-core positive women was only $14 \%$ (log rank test: $\chi_{\mathrm{df}=1}^{2}$ $=19.74 ; \mathrm{p}=0.001)$ (fig 1). This survival difference was also seen when early (stage 1) and late stage (stage 2 and more advanced) disease was compared (five year survival for $\beta$-core negative and early stage, $95 \%$; median survival for $\beta$-core negative and late stage, $37 \%$; no survivors at one year for $\beta$-core positive and early stage, median survival eight months; median survival for $\beta$-core positive and late stage, $12 \%$; log rank test: $\chi_{\mathrm{df}=3}^{2}=28.52 ; \mathrm{p}=$ 0.001 ) (fig 2). Women with squamous lesions also showed a significant survival advantage in the $\beta$-core negative group (two and four year survival for $\beta$-core negative women $79 \%$, $v$ 
$30 \%$ in $\beta$-core positive women (log rank test: $\left.\chi_{\mathrm{df}=1}^{2}=14.2 ; \mathrm{p}=0.001\right)$. Thirty four patients had $\beta$ hCG negative tumours on immunohistochemistry and 12 were positive. In the cases with positive immunohistochemistry, small numbers of tumour cells $(<5 \%)$ showed cytoplasmic staining. The assessment of $\beta$ hCG staining was not prognostic on its own and did not contribute to the significance of the urine $\beta$-core marker.

\section{RECURRENT DISEASE}

Nineteen women had negative urine $\beta$-core results at the time of presentation with recurrence and 19 were positive. The $\beta$-core values at the time of presentation were of no prognostic significance. The percent $\beta$-core positivity $(50 \%)$ of this group was not significantly higher than that in the primary presentation group $\left(33 \% ; \chi_{\mathrm{df}=1}^{2}=2.6 ; \mathrm{p}=0.1\right)$. Nineteen cases were $\beta$ hCG negative by immunohistochemistry from material collected at the time of the recurrence and 10 were positive. In this group, immunohistochemistry was of no prognostic significance, either alone or in combination with $\beta$-core.

\section{Discussion}

Our previous study ${ }^{11}$ showed that $\beta$-core is a useful short term prognostic marker in primary cervical cancer (18 months of follow up). Our present report now extends and confirms these findings with a larger group and longer follow up period. This is particularly important in cervical carcinoma, as most recurrences (treatment failure and persistent disease) will present within the first two years ${ }^{13}{ }^{14}$ and so may have been missed in the earlier study.

In the group of women who had recurrent disease, $\beta$-core was of no prognostic value. As the recurrent group is a selected subset with a very poor outcome, the higher number of positives is not unexpected. The phenomenon may be related to alterations in tumour biology associated with advanced disease or it may be attributable to previous treatment, for example radiotherapy. Reduced vascularity of irradiated areas containing tumour may have led to reduced amounts of $\beta$-core in the urine. In this study it was not possible to determine whether patients who were $\beta$-core positive at recurrence had been persistently $\beta$-core positive or whether this was a new phenomenon associated with disease recurrence and poor outcome. Cell culture has shown a positive growth effect of free $\beta$ hCG on cervical cancer cell lines (Crawford RAF, unpublished data) suggesting that a change in the local environment may lead to increasing aggressiveness. The five year survivors in the recurrent disease group were mostly those who had had pelvic exenteration.

In contrast to the earlier study, ${ }^{11}$ there was no difference in outcome related either to menopausal status or to age (above or below 40 years). The presence of high levels of luteinising hormone $\beta$-core fragment in postmenopausal urine $\mathrm{e}^{1516}$ and its cross reactivity with the antibody to $\beta$-core derived from $\beta$ hCG has been accounted for by using cut off limits appropriate for age and menopausal status.
The urine samples of 11 of the 57 patients with primary cancer and four of the 42 patients with recurrent cancer were too dilute for $\beta$-core analysis. This may have introduced bias, but as the samples were analysed in batches it was not possible to collect further specimens from these patients.

A tissue specimen is always taken at the time of diagnosis of primary and recurrent disease. The presence of $\beta$ hCG can be detected using immunohistochemistry and might obviate the need for a urine test ( $\beta$-core). However, in the present study $\beta$ hCG staining was of no prognostic value in any group. This might be related to technical problems, although we used an appropriate antigen retrieval method. ${ }^{12}$ The more likely explanation is that $\beta$ hCG is released rapidly following synthesis with little or no storage in the cell, and a random section of the tumour will be unlikely to contain more than a few positive cells at most. The small amount of staining in cases which were counted as positive is in contrast to positive transitional cell bladder carcinomas which show widespread staining. This suggests that the positive areas might represent non-specific staining. In colorectal cancer, the prognostic significance of raised serum $\beta$ hCG as a tumour marker was significant for poor outcome ${ }^{8}$ while immunohistochemical staining was of no value. ${ }^{17}$ These findings are reflected in our study.

The expression of $\beta$ hCG is stage related. ${ }^{4}$ Two of the three women who were $\beta$-core positive in the early stage subset of the primary group had involved pelvic lymph nodes. Therefore, larger numbers are required to determine whether the $\beta$-core expression and poor outcome are related to a larger tumour volume rather than to more aggressive disease. With the increased effectiveness of the United Kingdom national cervical screening programme, there has been a considerable fall in mortality and a reduction in the frequency of advanced disease. ${ }^{18}$ In advanced stage disease, the treatment options are limited to radiotherapy with or without adjuvant chemotherapy. Only in early stage disease, however, is there a significant potential for treatment modification, with tailoring the extent of surgery to suit the individual case with its prognostic indicators. In the woman with an early stage cancer and negative $\beta$-core, consideration may be given to a fertility sparing type procedure if indicated. In those women with positive $\beta$-core, the use of chemo-radiotherapy rather than surgery may be more appropriate. A large prospective study of urinary $\beta$-core in early stage cervical cancer is needed to ascertain whether it can be used as an index for modifying treatment.

This study was supported by a grant from the Cancer Research Committee, St Bartholomew's Hospital. Technical help with the immunohistochemistry was provided by Mrs Suzanne Jordan. Statistical advice was given by Ms Janice Thomas, Queen Mary and Westfield College, University of London.

1 Campo E, Palacin A, Benasco C, et al. Human chorionic gonadotrophin in colorectal carcinoma; an immunohistochemical study. Cancer 1985:59:1611-16.

2 Iles RK, Jenkins BJ, Oliver RTD, et al. Beta human chorionic gonadotrophin in serum and urine. A marker for metastatic urothelial cancer. $\operatorname{Br} \mathcal{F}$ Urol 1989;64:241- 4. 
3 Sheaff MT, Martin JE, Badenoch DF, et al. $\beta-\mathrm{hCG}$ as a prognostic marker in adenocarcinoma of the prostate. $\mathscr{f}$ Clin Pathol 1996;49:329-32.

4 Cole LA, Tanaka A, Kim GS, et al. $\beta$-Core fragment ( $\beta$-core/UGF/UGP), a tumor marker: a 7 -year report. Gynecol Oncol 1996;60:264-7

5 Carter PG, Iles RK, Neven P, et al. Measurement of urinary beta core fragment of human chorionic gonadotrophin in women with vulvovaginal malignancy and its prognostic significance. Br f Cancer 1995;71:350-3.

6 Iles RK, Chard T. Human chorionic gonadotrophin expression by bladder cancers: biology and clinical potential as an indicator of poor prognosis. F Urol 1991;145:453-8.

7 Regelson W. Have we found the "definitive cancer" biomarker? Cancer 1995;76:1299-301.

8 Carpelan-Holmström M, Haglund C, Lundin J, et al. Independent prognostic value of pre-operative serum markers CA 242 , specific tissue polypeptide antigen and human chorionic gonadotrophin beta, but not of carcinoembryonic antigen or tissue polypeptide antigen in colorectal onic antigen or tissue polypeptide

9 Moutzouris G, Yannopoulos D, Barbatis C, et al. Is beta-human chorionic gonadotrophin production by transitional cell carcinoma of the bladder a marker of aggressive
disease and resistance to radiotherapy? Br f Urol 1993;72 907-9.

10 Wehmann RE, Blithe DL, Flack MR, et al. Metabolic clearance rate and urinary clearance of purified $\beta$-core. 7 Clin Endocrinol Metab 1989;69:510-17.
11 Carter PG, Iles RK, Neven $\mathrm{P}$, et al. The prognostic significance of urinary $\beta$ core fragment in premenopausal women with carcinoma of the cervix. Gynecol Oncol 1994;55:271-6.

12 Norton AJ, Jordan S, Yeomans P. Brief, high-temperature heat denaturation (pressure cooking): a simple and effective method of antigen retrieval for routinely processed tissues. F Pathol 1994;173:371-9.

13 van Nagell JR, Rayburn WR, Donaldson ES, et al. Therapeutic implications of patterns of recurrence in cancer of the uterine cervix. Cancer 1979,44:2354-61.

14 Look KY, Rocereto TF. Relapse patterns in FIGO stage 1B carcinoma of the cervix. Gynecol Oncol 1990;38:114-20.

15 Akar AH, Gervasi G, Blacker C, et al. Human chorionic gonadotrophin-like and $\beta$-core-like materials in postmenopausal urine. $\mathcal{F}$ Endocrinol 1990;125:477-84.

16 Iles RK, Lee CL, Howes I, et al. Immunoreactive $\beta$-core-like material in normal postmenopausal urine: human chorionic gonadotrophin or LH origin? Evidence for existence of LH core. F Endocrinol 1992;133:459-66.

17 Webb A, Scott-Mackie P, Cunningham D, et al. The prognostic value of CEA, beta hCG, AFP, CA 125, CA 19-9 and C-erb B-2, beta hCG immunohistochemistry in advanced colorectal cancer. Ann Oncol 1995;6:581-7.

18 Sasieni P, Cuzick J, Farmery E. Accelerated decline in cervical cancer mortality in England and Wales. Lancet 1995;346:1566-7. 